\title{
ORIGINAL ARTICLE Improved recovery from spinal cord injury in rats with chronic parvovirus serotype-1a infection
}

\author{
$\mathrm{J} \mathrm{Kjell}^{1}$, L Olson and MB Abrams ${ }^{2}$
}

Objectives: A vendor informed us that rats shipped to us and used by us in a spinal cord contusion injury experiment were infected by rat parvovirus type 1a (RPV-1a). Our aim was therefore to determine whether this infection may have altered locomotor recovery or tissue pathology.

Setting: Stockholm, Sweden.

Methods: We induced a moderate contusion injury of the spinal cord in rats received from an (unknown to us) RPV-1a-contaminated facility. We compared the hind limb locomotor function between RPV-1a-infected rats and non-infected controls with the same spinal cord lesions, obtained before (historical control), as well as after infection (future controls). Histologically, we assessed spinal tissue sparing, astrocyte reactivity and the amount of macrophages/activated microglia.

Results: RPV-1a-infected rats had significantly better hind limb locomotor recovery compared with both 'historical' and 'future' controls. We also observed significantly better tissue sparing and axonal sparing around the injury site, as well as significant reductions in macrophages/activated microglia and astrocyte reactivity in the spinal cords of RPV-1a-infected rats.

Conclusion: The results stress the importance of knowing the health status of animals used to study central nervous system trauma and support the notion that acquired infections, even if asymptomatic, may alter response to injury in mammals. Furthermore, the results demonstrate that virus infections may have positive effects on functional recovery after spinal cord injury and indicate that RPV-1a infection may be neuroprotective by dampening secondary damage.

Spinal Cord (2016) 54, 517-520; doi:10.1038/sc.2015.208; published online 22 December 2015

\section{INTRODUCTION}

Infections developing after central nervous system (CNS) injury, such as pneumonia, are known to restrict neurological recovery in patients and thus to constitute a source of outcome variation. ${ }^{1}$ Similarly, the health status at the time of CNS injury can also influence outcome. Pre-existing infections, including chronic asymptomatic infections, are generally considered detrimental to recovery. ${ }^{2,3}$ The deleterious effects have primarily been attributed to the ability of infectious agents to modulate the inflammatory response, even when the infection is asymptomatic. Nevertheless, experimental research has repeatedly demonstrated that altering the inflammatory response after CNS injury may alter recovery and outcome both positively and negatively. ${ }^{4}$

Rat parvovirus type 1a (RPV-1a) is a non-enveloped DNA virus of the Parvoviride family, found in laboratory and wild rats, and causes a chronic 'asymptomatic' infection. ${ }^{5}$ Notably, RPV-1a infection is asymptomatic even in pups and severely immunocompromised rats, ${ }^{5,6}$ and no pathology or lesions are detected after experimental RPV-1a infection. ${ }^{5}$ It has been postulated that RPV-1a infection modulates immune function and that the presence of an RPV-1a infection in fact causes milder disease or delayed onset of clinical signs in tumor-bearing rats. ${ }^{5,7,8}$

We performed an experimental spinal cord injury study using a moderate contusion injury, unaware that our animals had been infected with RPV-1a before being delivered to us. Only post factum were we informed by the vendor that the rats shipped to us were infected by RPV-1a. This information was paralleled by our observations of unexpected improved spontaneous recovery from standardized weight drop spinal cord injury in these particular shipments of rats. Thus, we made it our objective to determine to what extent the RPV-1a infection had altered locomotor recovery or tissue pathology. At that time, non-infected controls of the same substrain were not available from the same vendor. We therefore compared our results from the RPV-1a-infected rats with two previous groups of rats obtained from the same vendor before the infection ('historical' controls). We later obtained re-derived, virus-free rats of the same strain from the same vendor and used these animals as a second control group ('future' controls). We also performed histological analysis of the injured cord, focusing on white matter sparing, axon sparing, macrophage load, astrocyte reactivity and cyst sizes.

\footnotetext{
Department of Neuroscience, Karolinska Institutet, Stockholm, Sweden

${ }^{1}$ Current address: Department of Physiological Genomics, Ludwig-Maximilians-Universität München (LMU), 80336 Munich, Germany.

${ }^{2}$ Current address: INCF, Karolinska Institutet, Stockholm 171 77, Sweden.

Correspondence: Dr J Kjell, Department of Neuroscience, Karolinska Institutet, Retzius väg 8, B2:4, Stockholm 171 77, Sweden.

E-mail: Jacob.kjell@ki.se

or Dr MB Abrams, INCF, Karolinska Institutet, Nobels väg 15A, Stockholm 17177, Sweden.

E-mail: Mathew@incf.org

Received 24 August 2015; revised 12 October 2015; accepted 18 October 2015; published online 22 December 2015
} 


\section{MATERIAL AND METHODS}

\section{Confirmation of parvovirus infection}

RPV-1a is transmitted through urine, feces and the oropharynx. ${ }^{5}$ When the RPV-1a infection was reported by the breeder (Scanbur, Sollentuna, Sweden), we independently re-confirmed the infection in selected rats sent to us (The National Veterinary Institute, SVA, Uppsala, Sweden). In a screen for seven common viral infections, 10 bacterial infections and two parasitic infections common to rats, the only positive finding was the presence of parvovirus (titer $>1: 20$ ) (Supplementary Figure 1).

\section{Surgery}

For all experiments, the same surgeon performed the spinal cord injuries. The moderate contusion injuries (Impactor, Keck Center for Neurosciences) were induced in female Sprague-Dawley rats $(N=14$ RPV-1a infected, $N=14$ historical controls, $N=8$ future controls; Scanbur) weighing $220-250 \mathrm{~g}$, as previously described in detail. ${ }^{9,10}$ 'Historical' controls consisted of experiments carried out 2-4 months before the studies of the RPV-la-infected animals, which in turn were conducted within 3 months of each other. The 'future' control group was studied 3 months after the substrain had been re-derived and cleared from the infection by the vendor.

\section{Open field locomotion}

The same individuals performed the behavioral analyses for all experiments. The Basso, Beattie, Bresnahan (BBB) locomotor rating scale was used to evaluate the hind limb locomotor function in an open field ${ }^{11}$ weekly for 8 weeks. Individual rats were observed by two experienced experimenters for $4 \mathrm{~min}$ to assess the hind limb locomotor function, which is scored from 0 to 21 (normal gait).

\section{Histochemistry}

At 56 days after injury, under deep anesthesia (Pentobarbital), the rats $(n=28)$ were transcardially perfused with $50 \mathrm{ml}$ Tyrode's solution containing $0.1 \mathrm{ml}$ of heparin (5000 IE ml-1), followed by $200 \mathrm{ml}$ fixative (4\% paraformaldehyde and $0.4 \%$ picric acid in $0.1 \mathrm{~m}$ phosphate-buffered saline). Spinal cords were dissected, post fixed for $1 \mathrm{~h}$ and transferred to $10 \%$ sucrose solution. Five consecutive $7 \mathrm{~mm}$ blocks of spinal cord, with the middle block covering the site of injury, were embedded in OCT media and frozen on dry ice. Twentymicrometer $(\mu \mathrm{m})$ sections were cut on a cryostat, thaw-mounted onto gelatincoated slides and further processed for immunofluorescence techniques and Luxol fast blue staining. A minimum of three sections per $7 \mathrm{ml}(\mathrm{mm})$ block was averaged from each animal when quantifying any histological marker.

Antibodies to CD68 (AbD Serotec, Kidlington, UK) were used to evaluate activated macrophages/microglia, glial fibrillary acidic protein antibodies (Dako, Stockholm, Sweden and Sigma-Aldrich, St Louis, MO, USA) to evaluate astrocyte reactivity and neurofilament-200 (NF-200) antibodies (Chemicon, Billerica, MA, USA) to visualize axons. Sections were incubated with primary antibody for $24 \mathrm{~h}(\mathrm{~h})$ at $4{ }^{\circ} \mathrm{C}$ and with fluorophor-labeled secondary antibodies (Alexa fluorophores, Molecular Probes, Waltham, MA, USA; Cy3, Jackson Laboratories, Sacramento, CA, USA) for $1.5 \mathrm{~h}$ at room temperature. Bound antibodies were visualized using epifluorescence microscopy (Nikon Eclipse 1000 , Surrey, UK) and photographed (Spot camera, Spot Advance software, Tekno optik, Tuttlingen, Germany) at $\times 5$ and $\times 10$. For Luxol fast blue staining, cryosections were stained with Luxol fast blue solution (solvent blue 17, 95\% ethyl alcohol and 5\% glacial acetic acid). Sections were then dehydrated in increasing concentrations of ethanol (70, 95 and 99.5\%) and mounted (Entellan, VWR International, Stockholm, Sweden).

\section{Analysis and statistics}

Image analysis was performed using an image-processing program (FIJI, www.fiji.sc). The intensity of immunoreactivity was measured in equal-sized boxes from a minimum of three sections per $7 \mathrm{~mm}$ block/animal. For comparisons of $\mathrm{BBB}$ scores, repeated measures analysis of variance was performed with the Bonferroni post-hoc test. The confidence interval was set at $95 \%$. Data are presented as mean \pm s.e.m. Area and intensity measures were compared using Student's $t$-test and presented as mean \pm s.e.m. $P$-values $<0.05$ were considered significant.

\section{Statement of ethics}

We certify that all applicable institutional and governmental regulations concerning the ethical use of animals were followed during the course of this research. All animal work was approved by the Northern Stockholm Animal Ethics Committee.

\section{RESULTS}

We found that the hind limb function recovered better in rats with chronic parvoviral infection when compared with both 'historical' and 'future' controls. Infected rats achieved the BBB score 7 already 2 weeks after injury, whereas it took 6 weeks for uninfected rats to achieve the same score (Figure 1a). Eight weeks after injury the locomotor function among infected rats ranged from weight support in stance to weight-supported dorsal and plantar stepping, whereas among uninfected rats the locomotor function ranged from extensive movement of all three joints in the hind limb to sweeping movements without weight support. This resulted in final average BBB scores of $9.1 \pm 0.4$ compared with $7.4 \pm 0.5$ for RPV-1a-infected rats $(n=14)$ and historical controls $(n=14)$, respectively. The 'future' control rats (re-derived and uninfected) had similar locomotor recovery and regained similar levels of the locomotor function at the end of the experiment (final BBB score was $7.6 \pm 0.3$ ) as were obtained in the 'historical' controls.

Histological evaluation 8 weeks post injury revealed that the spinal cords of infected rats had a significantly larger amount of spared white matter compared with those of uninfected rats $(\mathrm{F}=4.40, P=0.016)$ (Figures $1 \mathrm{~b}-\mathrm{d}$ ). Although quantification of area occupied by spinal cysts within the injury site was difficult, because of the presence of collapsed cysts, the cyst area tended to be smaller in spinal cords of infected rats (data not shown). We also observed a significantly larger amount of NF-200 immunoreactivity $(\mathrm{F}=1.76, P=0.012)$ in the spinal cords of infected, compared with uninfected rats (Figures $1 \mathrm{e}-\mathrm{g}$ ).

Spinal cords of infected rats had significantly lower CD68 immunoreactivity within the injury site at 8 weeks post injury compared with historical controls $(t=4.094, P<0.01)$ (Figure 2). We also observed that the mean intensity of CD68 immunoreactivity was lower in the spinal cord 7-14 $\mathrm{mm}$ rostral, as well as caudal to the injury site in infected rats (Figures $2 \mathrm{a}-\mathrm{c}$ ). In addition, the intensity of glial fibrillary acidic protein immunoreactivity was significantly less within the injured area $(\mathrm{F}=2.24, P=0.0085)$ in $\mathrm{RPV}-1 \mathrm{a}$-infected rats, compared with the historical controls (Figures 2d-f).

\section{DISCUSSION}

Here, we report that chronic RPV-1a infection alters recovery from contusion spinal cord injury in rats. We have previously demonstrated substrain differences in spontaneous recovery of function after spinal cord injury. ${ }^{10}$ The Scanbur substrain used in this study had over the course of several previous experiments proven to reach a particular, reproducible level of recovery from a carefully defined moderate contusion injury, such that hind limbs typically do not reach the ability to support body weight. ${ }^{12}$ When, in later studies, we found that the same substrain, obtained from the same vendor and subjected to the same standardized weight drop injury to the spinal cord, began to recover better, we went to great length attempting to detect whether this could be explained by alterations of routines or equipment in our lab. We purchased a new weight drop apparatus of the same type we already had, but the results remained the same. When the vendor finally, and post factum, informed customers that their rats had been 

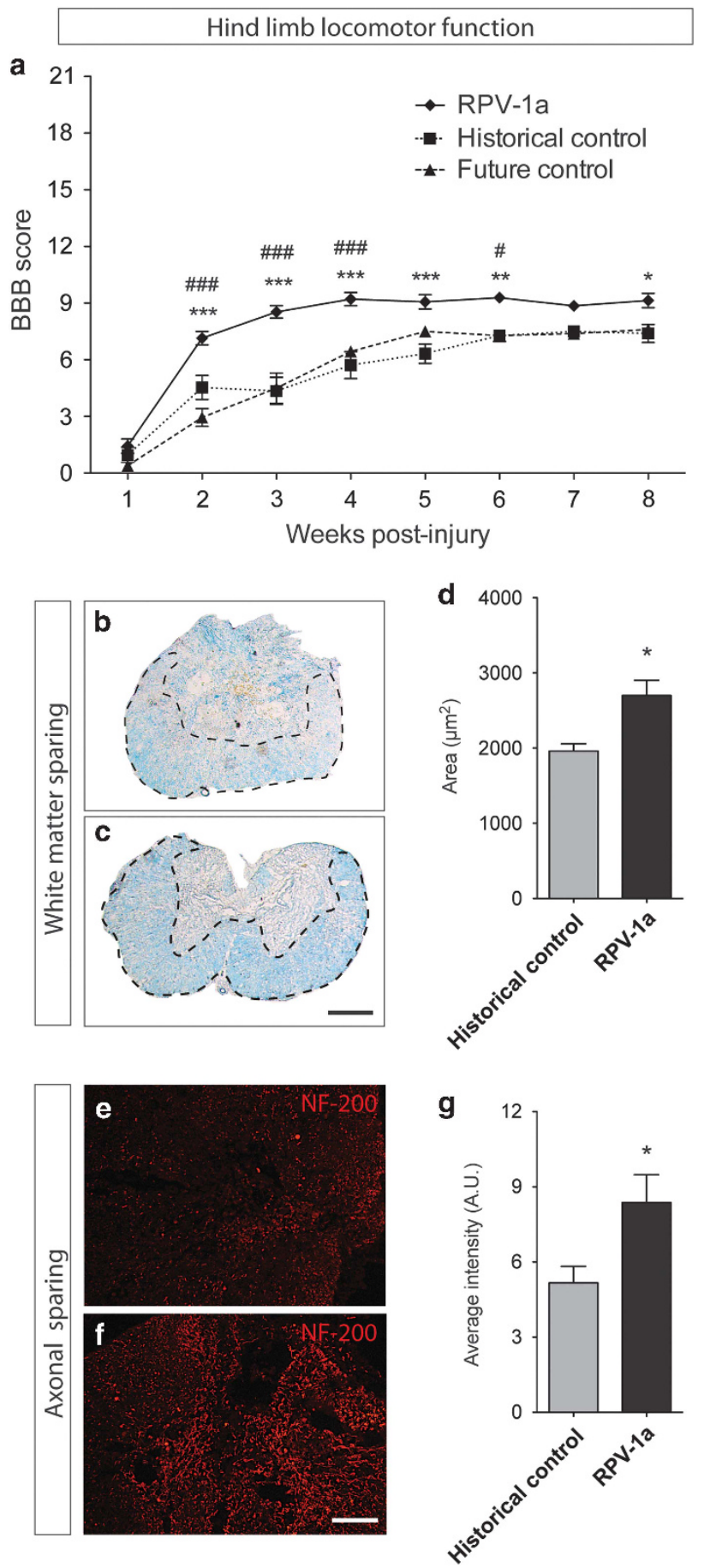

Figure 1 RPV-1a infection improves hind limb locomotor recovery and tissue/axonal sparing. (a) BBB scores of the hind limb locomotor function across an open field during 8 weeks after injury. RPV-1a-infected rats vs historical $\left(^{*}\right)$ and future (\#) controls. Historical controls were studied before receiving the RPV-1a-infected rats, and the future controls after this particular substrain had been re-derived and cleared from the infection. Data presented as mean \pm s.e.m. ${ }^{* / \#} P<0.05,{ }^{* *} P<0.01$ and ${ }^{* * * / \# \# \#} P<0.001$. (b, c) Representative micrographs of luxol fast blue stained spinal cord sections at the injury site week 8 post injury. Scale bar, $250 \mu \mathrm{m}$. (b) Historical controls (uninfected), (c) RPV-1a infected. (d) Quantification of the area of the spinal cord at the injury site. (e, f) Representative micrographs of NF-200 immunoreactivity within the injury site at week 8 post injury. (e) Historical controls (uninfected), (f) RPV-1a infected. (g) Quantification of NF-200 immunoreactivity within the injury site of the spinal cord. Mean \pm s.e.m. ${ }^{*} P<0.05$. Scale bar, $200 \mu \mathrm{m}$.
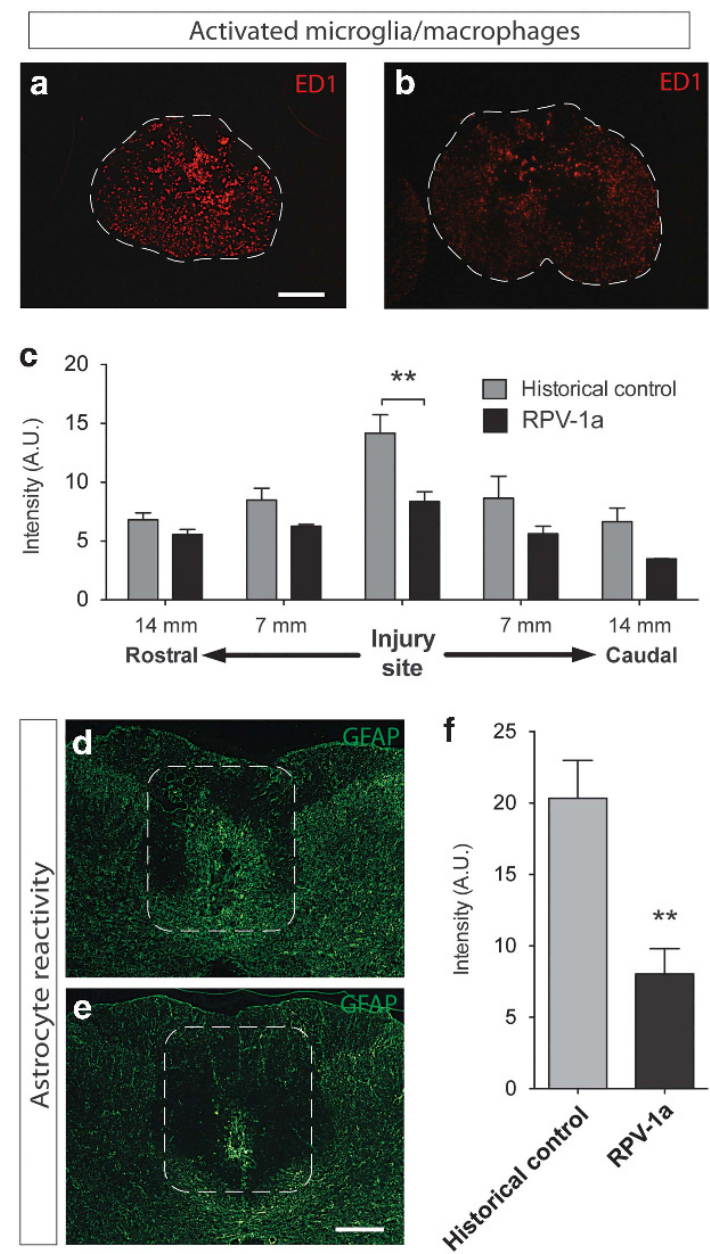

Figure 2 RPV-1a infection dampens the inflammatory response to spinal cord damage. Representative micrographs of CD 68 immunoreactivity within the injury site week 8 post injury. Scale bar, $400 \mu \mathrm{m}$ (a) Historical controls (uninfected). (b) RPV-1a infected. (c) Quantification of CD 68 immunoreactivity at different levels of the injured spinal cord. (d, e) Representative micrographs of glial fibrillary acidic protein immunoreactivity caudal to the injury site on week 8 post injury. (d) Historical controls (uninfected), (e) RPV-1a infected. (f) Quantification of glial fibrillary acidic protein immunoreactivity in the spinal cord. Scale bar, $50 \mu \mathrm{m}$ Data presented as the mean \pm s.e.m. ${ }^{* *} P<0.01$.

carrying chronic RPV-1a infection during the period when we noted improved recovery, we decided to compare 'historical' and 'future' controls (re-derived virus-free animals) of the same substrain from the same vendor with the RPV-1a-infected rats. This allowed us to conclude that locomotor recovery was markedly accelerated and final locomotor outcome moderately improved in RPV-1a-infected rats. These robust improvements of the locomotor function correlated to significantly lesser loss of spinal cord tissue and neurofilamentimmunoreactive fibers within the injury site. We also found a marked reduction in inflammation at the site of injury in RPV-1a-infected rats, suggesting that RPV-1a affected the inflammatory response. In this study, all experiments were performed by the same researcher, and the same individuals assessed the locomotor function of all groups. Hence, we believe we have found a real parvoviral effect on pathology and functional outcome, as opposed to an artifact due to differences in procedure. Furthermore, our experience using this rat substrain tells us that uninfected rats would not display such great acceleration in 
locomotor recovery, nor such great reduction in inflammation, as displayed by the infected rats. Thus, our data indicate the importance of knowing the health status of animals used in experimental spinal cord injury research, as pathogens capable of modulating the immune response may potentially mask or exacerbate the effects of experimental therapies.

Many infectious agents can modulate the immune response but are rarely investigated for their potential therapeutic properties, although there are examples such as parasite infection in MS patients. ${ }^{13}$ Nevertheless, many experimental interventions targeting the immune response have been reported to significantly enhance functional outcome and reduce tissue pathology in CNS injuries. ${ }^{4,14,15}$ RPV-1a DNA has been found in various immune organs, such as bone marrow, liver, spleen, lymphoid tissue and thymus, ${ }^{16}$ and has been reported to modulate immune function. ${ }^{5}$ Our findings of improved recovery from spinal cord injury in rats carrying the RPV-1a virus lend further support to the notion that certain infections may modulate the immune response in response to CNS injury toward a more favorable outcome. It remains to be demonstrated whether there are human virus infections capable of improving outcome after spinal cord injury.

The robust effects of an ongoing, subclinical RPV-1a infection on structural and functional recovery from spinal cord injury in rats suggest that acquired infections, even when asymptomatic, may similarly contribute to interindividual differences in patients' recovery from CNS injury and that understanding the underlying mechanisms may have therapeutic implications.

\section{DATA ARCHIVING}

There were no data to deposit.

\section{CONFLICT OF INTEREST}

The authors declare no conflict of interest.

\section{ACKNOWLEDGEMENTS}

We thank Simone Codeluppi for valuable advice and Karin Pernold for technical assistance. This study was supported by the Swedish Brain Foundation (MBA), the Swedish Research Council, Wings for Life, the Karolinska Institute (including a DPA award), the Karolinska StratNeuro initiative and the Swedish Agency for Innovation Systems (VINNOVA).
1 Failli V, Kopp MA, Gericke C, Martus P, Klingbeil S, Brommer B et al. Functional neurological recovery after spinal cord injury is impaired in patients with infections. Brain 2012; 135: 3238-3250.

2 Teeling JL, Perry VH. Systemic infection and inflammation in acute CNS injury and chronic neurodegeneration: underlying mechanisms. Neuroscience 2009; 158: 1062-1073.

3 Ousman SS, Kubes P. Immune surveillance in the central nervous system. Nat Neurosci 2012; 15: 1096-1101.

4 David S, Kroner A. Repertoire of microglial and macrophage responses after spinal cord injury. Nat Rev Neurosci 2011; 12: 388-399.

5 Ball-Goodrich LJ, Leland SE, Johnson EA, Paturzo FX, Jacoby RO. Rat parvovirus type 1: the prototype for a new rodent parvovirus serogroup. J Virol 1998; 72: 3289-3299. 6 Jacoby R. Parvovirus infections of mice and rats. Semin Virol 1995; 6: 329-337.

7 McKisic MD, Lancki DW, Otto G, Padrid P, Snook S, Cronin DC et al. Identification and propagation of a putative immunosuppressive orphan parvovirus in cloned $\mathrm{T}$ cells. J Immunol 1993; 150: 419-428.

8 Jacoby R, Ball-Goodrich L. Rodent parvovirus infections. Lab Anim Sci 1996; 46: 370-380.

9 Gruner JA. A monitored contusion model of spinal cord injury in the rat. J Neurotrauma 1992; 9: 123-128.

10 Kjell J, Sandor K, Josephson A, Svensson Cl, Abrams MB. Rat substrains differ in the magnitude of spontaneous locomotor recovery and in the development of mechanical hypersensitivity after experimental spinal cord injury. J Neurotrauma 2013; 30: 1805-1811.

11 Basso DM, Beattie MS, Bresnahan JC. A sensitive and reliable locomotor rating scale for open field testing in rats. J Neurotrauma 1995; 12: 1-21.

12 Abrams MB, Nilsson I, Lewandowski SA, Kjell J, Codeluppi S, Olson L et al. Imatinib enhances functional outcome after spinal cord injury. PLOS ONE 2012; 7: e38760.

13 Correale J, Farez MF. The impact of parasite infections on the course of multiple sclerosis. J Neuroimmunol 2011; 233: 6-11.

14 Gensel JC, Kigerl KA, Mandrekar-Colucci SS, Gaudet AD, Popovich PG. Achieving CNS axon regeneration by manipulating convergent neuro-immune signaling. Cell Tissue Res 2012; 349: 201-213.

15 Bethea JR, Dietrich WD. Targeting the host inflammatory response in traumatic spinal cord injury. Curr Opin Neurol 2002; 15: 355-360.

16 Ueno Y, Sugiyama F, Sugiyama Y, Ohsawa K, Sato H, Yagami K. Epidemiological characterization of newly recognized rat parvovirus, "rat orphan parvovirus". J Vet Med Sci 1997; 59: 265-269.

(1)(2) (2) This work is licensed under a Creative Commons Attribution-NonCommercial-ShareAlike 4.0 International License. The images or other third party material in this article are included in the article's Creative Commons license, unless indicated otherwise in the credit line; if the material is not included under the Creative Commons license, users will need to obtain permission from the license holder to reproduce the material. To view a copy of this license, visit http://creativecommons.org/licenses/bync-sa/4.0/

Supplementary Information accompanies this paper on the Spinal Cord website (http://www.nature.com/sc) 